\title{
Solution of TSP Problem of Measurement of Soil Attributes for Precision Agriculture
}

\author{
Zehui Yun, Shiquan Shao*, Tongning Mai \\ College of Electrical \& Information Engineering, Southwest Minzu University, Chengdu, China \\ Email: $18428385947 @ 163 . c o m$
}

\begin{abstract}
There are many PATCHES in the measurement of soil attributes in precision agriculture, which are greatly different from the surrounding soil attributes. In this paper, firstly, we build a TSP model of precision agriculture based on the location of PATCHES. Then, we use the improved ant colony algorithm to solve the TSP model. Numerical simulations show the effectiveness and reliability of the proposed method.
\end{abstract}

Keywords: Precision agriculture, TSP problem, ant colony algorithm

\section{$1 \quad$ Introduction}

Precision agriculture is based on information technology, which implements a set of modern agricultural operation and management system in accordance with spatial variability, positioning, timing and quantification. To achieve precise control, we must know the specific situation of each location in order to adapt to local conditions. In fact, multiple PATCHES appear because of the spatial and temporal variability of soil, such as soil type, nutrient availability and crop pests. Therefore, it is necessary to survey the whole land. However, except for the location of PATCHES, the other soil conditions can be calculated from the data of nearby locations. Therefore, we only need to measure the soil condition of these PATCHES. If some prior information is available (the location of these PATCHES is known in advance), then the robot can choose key, informative locations to obtain the measurements from. Furthermore, if nearby points are correlated with the robot need not visit a location exactly, but instead visit some point in its neighborhood. We can reduce the total time by combining measurement locations of nearly points with overlapping neighborhoods. This leads to a novel variant of the classical Euclidean traveling salesperson problem (TSP), which we think the traveling salesperson problem in precision agriculture.

TSP problem is one of the famous problems in the field of mathematics. Many domestic and overseas scholars adopt different methods to solve the TSP problem [1-9]. For TSP problems in precision agriculture: Thomas C. Thayer and other scholars in the literature [10] proposed that the machine should be adjusted effectively according to the battery power and usage time in precise irrigation. Thomas C. Thayer and other scholars also make a feasibility analysis on the application of two greedy heuristic methods in this problem. In the literature [11], S and K use unmanned ground vehicles and unmanned aerial vehicles to quickly get soil maps. They also improved algorithms for unmanned ground vehicles and unmanned aerial vehicles. S.T.Jawaid and S.L.Smith scholars used greedy algorithms to extend the Hamiltonian cycle of the classical maximum weight to solve this problem[12]; Yasmina Bestaoui and Said Mammar applied genetic algorithm to obtain the shortest path in agricultural environment with depression obstacles, and verified the effectiveness of the method in MATLAB[13]. In the literature [14], Zhou K was to develop a planning method that generated a feasible area coverage plan for agricultural machines executing non-capacitated operations in inhabiting multiple obstacle areas, which was transformed into the TSP problem, and the effectiveness of the method was verified through practical experiments. However, none of the above documents considers the shortest path problem of ridge tillage farmland.

Therefore, the purpose of this paper is to find the optimal path in ridge tillage farmland. This paper intends to use ant colony algorithm to solve TSP problem in precision agriculture and obtain the optimal path. First, we need to get the location of PATCHES. Then the model is built according to the condition of these PATCHES, and turns the problem into TSP problem in precision agriculture. Finally, ant colony algorithm is used to solve the TSP problem. 


\section{The TSP Problems in Precision Agriculture}

The TSP problem is generally described as: A traveler visits $\mathrm{N}$ cities, each of which must be visited only once, and needs to find a shortest route that includes all $\mathrm{N}$ cities. Its mathematical model is as follows [15]:

An access order for cities $V=\left\{v_{1}, v_{2}, \ldots, v_{n}\right\}$ is $T=\left(t_{1}, t_{2}, \ldots, t_{n}\right)$, where $t_{i}=V(i=1,2, \ldots, n)$, and $t_{n+1}=t_{1}$, then the problem is to find $\min \sum_{\mathrm{T} \in \Omega_{\mathrm{i}}=1}^{\mathrm{n}} \mathrm{d}_{\mathrm{t}_{\mathrm{i}} \mathrm{t}_{\mathrm{i}+1}}$, where $\Omega$ is all the possible routes that the $\mathrm{N}$ cities do not repeat.

We make enumerative measurements of fields and map soil moisture based on the results (Fig. 1). As shown in Fig. 1, the figure is a map of soil moisture, which is divided into 10 grades according to the color depth to distinguish the moisture situation in the soil. Simplify it to get a two-dimensional map, as shown in Fig.2. In Fig. 2, the black pattern represents PATCH, and the black line represents the crop area. Most of the farmland is FENG or TIAN, and the crop area machines can't pass, thus the TSP problem in precision agriculture has come into being.

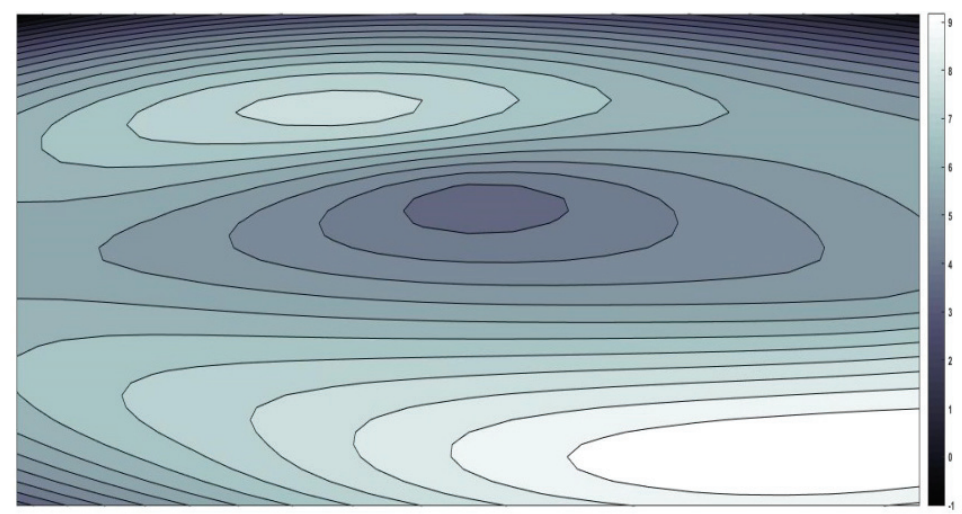

Figure 1. Map of soil moisture

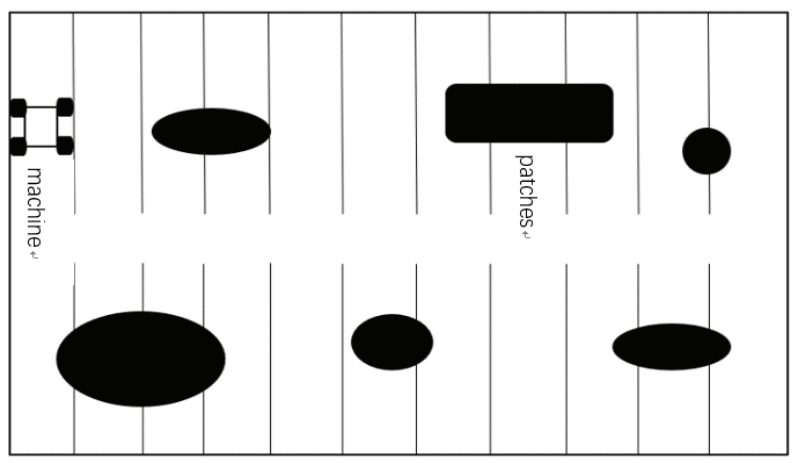

Figure 2. PATCH diagram

\section{Ant Improved Colony Algorithm for Solving the TSP Problem in Precision Agriculture}

If there are $\mathrm{M}$ ants, each ant chooses the next PATCH according to the probability function which takes the PATCH distance and the number of pheromones on the link edge as variables (if $\tau_{i j}(t)$ is the $e(i, j)$ pheromone intensity at time $\mathrm{t}$ ). The PATCH distance can be divided into three categories according to the actual situation of the field. As shown in Fig. 3 (The white blank area represents the ridge area allowing machines to pass through, black areas represent crop areas machines are not allowed to pass): 


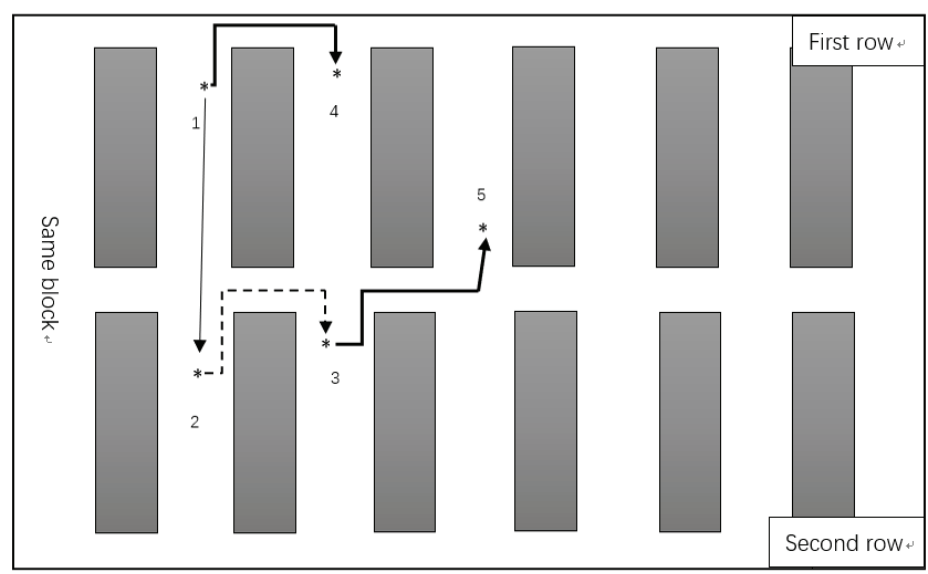

Figure 3. PATCH distance diagram

(1) When two PATCHES are located in the same area(as shown in Fig. 3, where the ' 1 and 2'), the formula of the shortest distance between two points is as follows:

$$
\mathrm{L}=\sqrt{\left(x_{1}-x_{2}\right)^{2}+\left(y_{1}-y_{2}\right)^{2}}
$$

(2) When two PATCHES belong to the area of the first row or the second row(as shown in Fig. 3, '1, 4, or 2,3 '), solving the shortest distance in this case, we need to compare the shortest distance between the edge ridge and the middle ridge. The result of comparison is the shortest path, the formula of the shortest distance between the two points is as follows:

$$
\mathrm{L}=\min \sum_{i \in 1,2}^{2} \sqrt{\left(h_{i}-y_{1}\right)^{2}+\left(h_{i}-y_{2}\right)^{2}+\left(x_{1}-x_{2}\right)^{2}}
$$

where $h_{1}$ is the coordinate value of the edge field; $h_{2}$ is the coordinate value of the middle field.

(3) When two PATCHES are located in the first and second row and do not belong to the same PATCH area (as shown in Fig. 4, the '3, 5' PATCH), because it belongs to the "one up and down" two rows, the middle distance is the shortest, then the two-point shortest distance formula is

$$
\mathrm{L}=\sqrt{\left(x_{1}-x_{2}\right)^{2}}+\sqrt{\left(y_{1}-h_{z}\right)^{2}}+\sqrt{\left(y_{2}-h_{z}\right)^{2}}
$$

where $h_{z}$ is the coordinate value of the middle field.

It is stipulated that ants are not allowed to transfer to the visited PATCHES unless the tour is completed, which is controlled by taboo tables. $\left(\operatorname{tabu}_{\mathrm{k}}\right.$ indicates the taboo list of the $\mathrm{k}$ ant, $\operatorname{tabu}_{\mathrm{k}}(\mathrm{s})$ indicate the $\mathrm{s}$ element in the taboo list). After completing the tour, the ant leaves a pheromone on each side of its visit. At the initial moment, the pheromone on each path is equal, if $\tau_{i j}(t)=C$ (C is a constant). During the movement, the direction of the transfer is determined according to the amount of information on each path. The rule that ant $k$ is selected from the PATCH $i$ to the next PATCH $j$ is: when $\mathrm{q} \leq \mathrm{q}_{0}$ :

$$
\mathrm{j}=\arg \max _{u \in \text { allowed }_{k}}\left\{\left[\tau_{i u}(t)\right]^{\alpha} \cdot\left[\eta_{i u}\right]^{\beta}\right\}
$$

when $\mathrm{q}>\mathrm{q}_{0}$, The ant selects the next point according to the transition probability formula:

$$
P_{i j}^{k}(t)=\left\{\begin{array}{c}
\frac{\left[\tau_{i j}(t)\right]^{\alpha}\left[\eta_{i j}\right]^{\beta}}{\sum_{s \in \text { allowed }_{k}\left[\tau_{i s}(t)\right]^{\alpha}\left[\eta_{i s}\right]^{\beta}},}, \mathrm{j} \in \text { allowed }_{k} \\
0 \quad \text { else } \\
\eta_{i j}=\frac{1}{d_{i j}}
\end{array}\right.
$$

where: $\mathrm{q}_{0}$ and $\mathrm{q}$ are the given constant between $(0,1) ; \tau_{\mathrm{iu}}(\mathrm{t})$ represents the pheromone concentration on the path between the PATCH $\mathrm{i}$ and the PATCH $\mathrm{u} ; \mathrm{d}_{\mathrm{ij}}$ is the distance between the two PATCHES; The amount of information accumulated by two ants in the course of movement is expressed in $\alpha$, which is the pheromone importance factor (the amount of residual information $\tau_{i u}(t)$ ); The relative importance of ants 
in guiding ant colony search in the course of movement is expressed in $\beta$, which is the heuristic function importance factor (expectation $\eta_{\mathrm{ij}}$ ); allowed $_{\mathrm{k}}$ denotes the set of the current feasible entry points of ant $\mathrm{k}$.

In the iteration process of traditional ant colony algorithm, if the new optimal path does not appear, the pheromone on the current optimal path will continue to increase under the effect of update strategy, which may lead to two disadvantages: For one thing, the pheromone on the current optimal path may be stagnant due to over-strengthening; For another, when the new optimal path appears, the pheromone intensity on the path may be much lower than the original optimal path, which will reduce the search efficiency. In view of the limitation of the above pheromone update method, the pheromone update method combining local update and global dynamic update is proposed.

(1) Local update

Ants use formula (7) to update pheromones on each side $(\mathrm{i}, \mathrm{j})$ in path construction.

$$
\tau_{i j}(\mathrm{t}+1)=(1-\varepsilon) \tau_{i j}(\mathrm{t})+\varepsilon \tau_{0}
$$

where: $\varepsilon$ is local trajectory persistence; $\tau_{0}$ is the initial value of the pheromone. The function of pheromone local update is that every time an ant passes through an edge, the pheromone on the edge will be reduced, which reduces the probability of other ants selecting the edge and increases the chance of exploring other edges.

(2) Global dynamic update

When an iteration is finished, the pheromone is updated globally by using the current optimal path formula (8) (9).

$$
\begin{gathered}
\tau_{i j}(\mathrm{t}+1)=(1-\rho) \tau_{i j}(\mathrm{t})+\rho \Delta \tau_{i j} \\
\Delta \tau_{i j}=\frac{L_{1}-L_{g}}{L_{g}}
\end{gathered}
$$

where: $\rho$ is a pheromone volatilization factor; $1-\rho$ is the degree of information disappearance; $L_{1}$ is the optimal iteration path length of the current iteration; $\mathrm{L}_{\mathrm{g}}$ is the current optimal path length; Update pheromone feedback on the current optimal path and keep it in the next iteration until there is a better path to replace it.

According to the description of the above improved strategy, the steps to summarize the improved algorithm are as follows:

Step1 Parameter initialization. Let the iteration counter $\mathrm{NC}=0$, set the current optimal path length $\mathrm{S}$, maximum number of iterations $\mathrm{T}$, distance between PATCHES $d_{i j}(\mathrm{i}, \mathrm{j}=1, \ldots, \mathrm{n})$, heuristic information $\eta_{i j}(\mathrm{i}, \mathrm{j}=1, \ldots, \mathrm{n})$, pheromones on the path $\tau_{i j}(\mathrm{i}, \mathrm{j}=1, \ldots, \mathrm{n})$.

Step2 Ant position initialization. Initialize the taboo table $t_{k}(\mathrm{k}=1,2, \ldots, \mathrm{m})$ of $\mathrm{m}$ ants, the length of the path to be travelled $L_{k}(\mathrm{k}=1,2, \ldots, \mathrm{m})$. All ants randomly select the initial PATCH, add the selected PATCH to the taboo table $t_{k}$, and update the value of $L_{k}$.

Step3 Path construction. Each ant chooses its path according to formula (5), adds the selected PATCHES to $t_{k}$, and updates the value of $L_{k}$.

Step4 Pheromone local update. For each PATCH selected by ants, pheromone local updates of the path just passed (i,j) are performed according to formula (7).

Step5 Pheromone global dynamic update. Statistical analysis of the current optimal path, comparing $L_{k}$ with $\mathrm{S}$. If $L_{k}<S, \mathrm{t}$ replace $t_{k}$. According to formulas (8) and (9), the pheromone of the current optimal path is updated globally and dynamically.

Step6 Iterative loop. If $\mathrm{NC} \leq \mathrm{T}$, return to step2 and start a new iteration, otherwise the algorithm end and output the optimal path length and the optimal path.

\section{$4 \quad$ Experimental Results and Analysis}

In order to verify the feasibility of the above algorithm, the improved ant colony algorithm for TSP in precision agriculture was simulated and verified by using software MATLAB. The field model shown in Fig. 4 is obtained by standardizing the coordinates of the single-row eggplant field, e.g. the plant spacing is 40 
$\mathrm{cm}$ and the row spacing is $60 \mathrm{~cm}$. The patch coordinates in the simulation examples are randomly generated by the system, totaling 22. In practice, there will be no PATCHES on the land too close to each other. For this reason, we should adjust these similar points, and fine-tune the coordinates of some points that appear in the crop area to remove these points from the crop area. Using MATLAB 2017a to simulate, run on i5-4460 3.2GHZ, 10GB memory computer, the experimental parameters are set as follows:

$$
\mathrm{m}=35, \mathrm{~N} C_{\max }=50, \alpha=1, \beta=5, \rho=0.1, Q=100
$$

where $\mathrm{m}$ is the number of ants, $\mathrm{NC}_{\max }$ represents the maximum number of iterations, $\alpha$ represents the pheromone importance factor, $\beta$ represents the heuristic function importance factor, $\rho$ represents pheromone volatilization factor, $\mathrm{Q}$ represents the total amount of pheromone released.

The path corresponding to the operation results is shown in Fig. 4. From the graph, it can be clearly seen that from a PATCH, each PATCH visits once, after traversing all PATCHES, returns to the starting point and finds the shortest path. The row spacing and crop spacing in the map are based on the actual row spacing and crop spacing of eggplant planting; the gray area is crop area (machine can't pass), and the white area is ridge area (machine can pass).

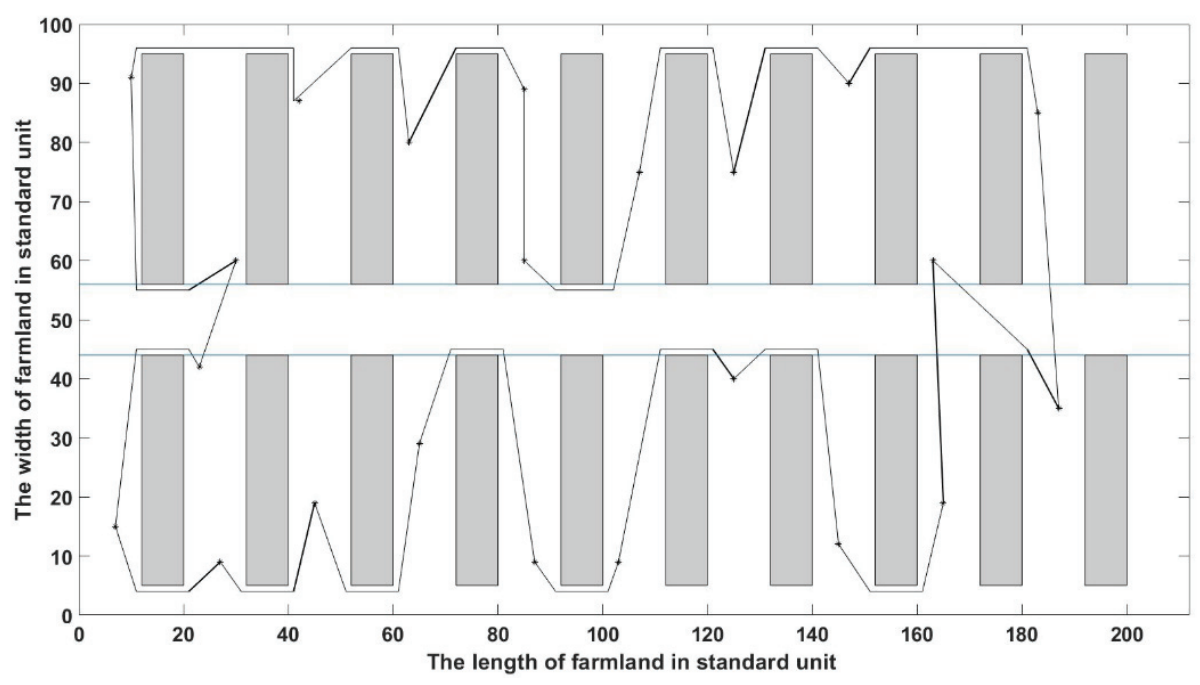

Figure 4. TSP roadmap

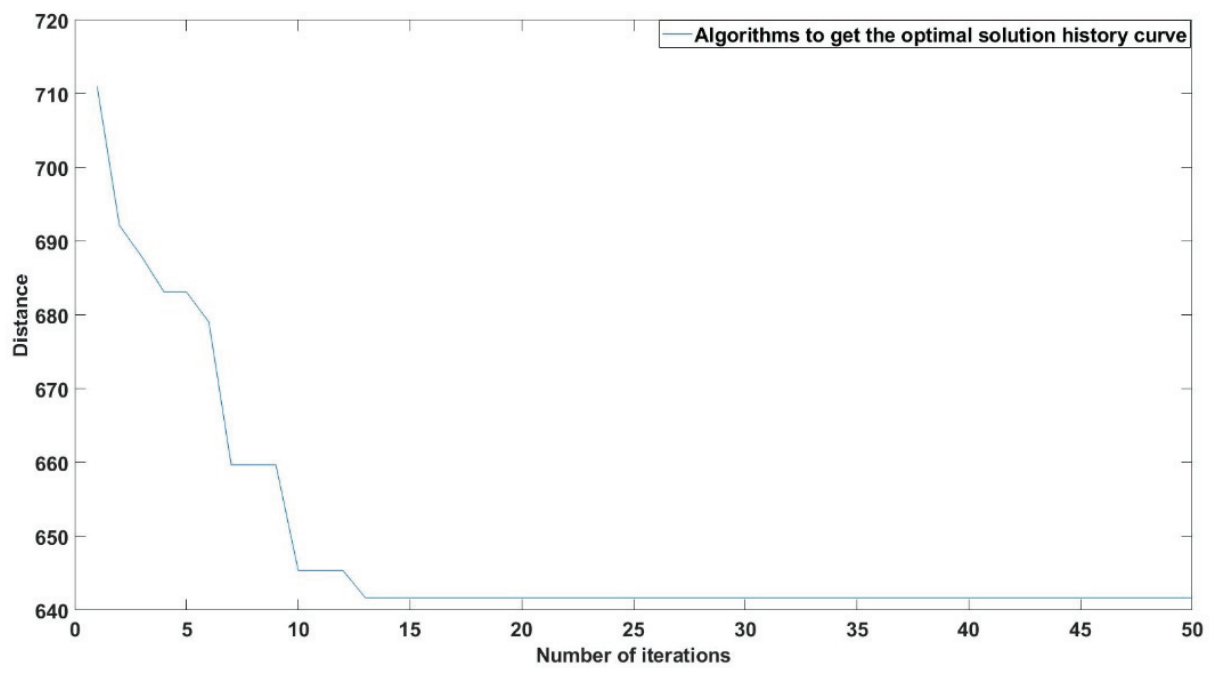

Figure 5. Algorithms to get the optimal solution history curve 


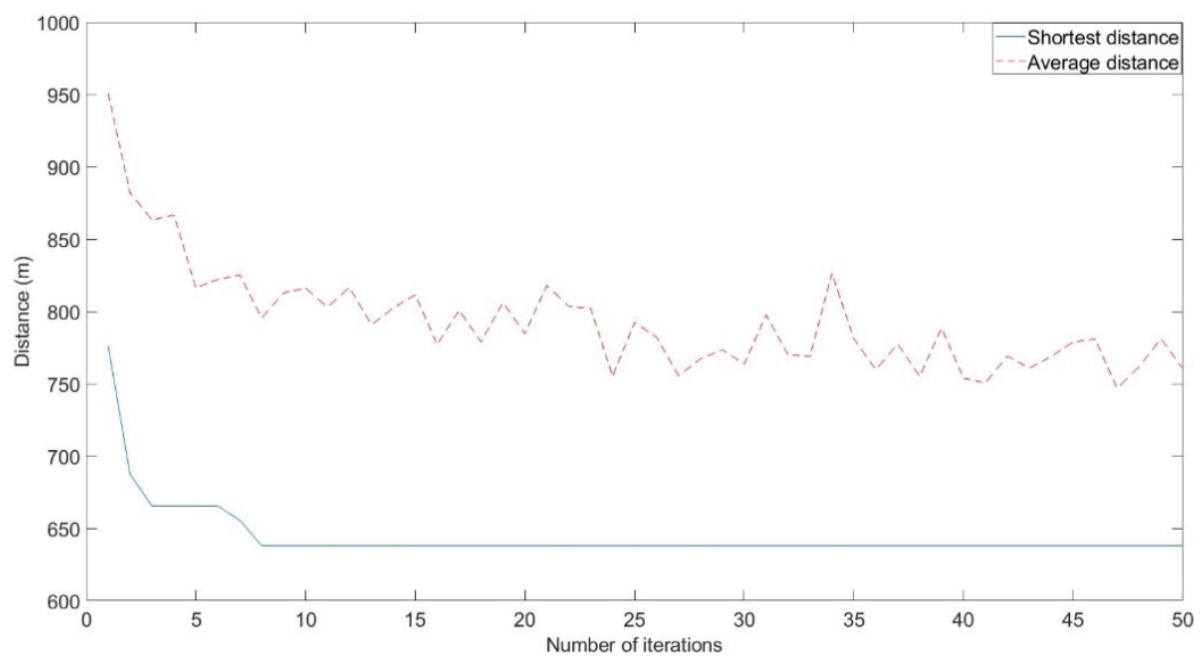

Figure 6. Comparison of the shortest distance and average distance for each iteration

Fig. 5 shows the relationship between the number of iterations and the shortest distance. As the number of iterations increases, the shortest distance shows a downward trend. When the number of iterations is more than 13, the shortest distance not change anymore, which indicating that the best path has been found.

Fig. 6 shows the relationship between the shortest distance and the average distance. As the number of iterations increases, both the shortest distance and the average distance show a downward trend. When the number of iterations is more than 13, the shortest distance does not change, but the average distance still fluctuates in a small range.

The simulation results verify the mathematical model of TSP problem of measurement of soil attributes for precision agriculture and the effectiveness of the algorithm. The simulation results show that the improved ant colony algorithm can quickly find the optimal path for TSP problem of measurement of soil attributes for precision agriculture, which can effectively reduce fuel and save running time.

\section{Conclusion}

In the soil measurement of precision agriculture, spatial and temporal variability of soils can lead to PATCHES. The problem of measuring soil moisture in these PATCHES by machine is transformed into TSP problem. The improved ant colony algorithm is used to solve the problem. Compared with the traditional, this ant colony algorithm uses the local and global update method of pheromone, improves the search efficiency and avoids falling into the local optimum. The simulation results show that the algorithm can not only reduce the running distance of the machine effectively, but also saving fuel and time.

Acknowledgements. The work of this paper is supported by the Southwest Minzu University Graduate Innovative Research Project (Master Program CX2018SZ95). A special acknowledgement should give to Southwest Minzu University for its experimental conditions and technical support.

\section{References}

1. LI Chengbing, GUO Ruixue, LI Min. Application of improved ant colony algorithm in travelling salesman problem[J]. Journal of Computer Applications,2014(z1).

2. Wang Peidong, Tang Gongyou, Yang Xixin, et al. An Improved Ant Colony Algorithm for Traveling Salesman Problems [J]. Periodical of Ocean University of China, 2013, 43(1):93-97.

3. Yang Xuefeng. Ant Colony Algorithm for TSP Problem [D]. Jilin University,2010.

4. Xu Kaibo. Improvement of Ant Colony Optimization Algorithm and Its Applications in Several Optimization Problems [D]. Jiangnan University,2018. 
5. YU Ying-ying, CHEN Yan, LI Tao-ying, et al. Improved genetic algorithm for solving TSP [J]. Control and Decision,2014(8).

6. SHEN Xuanjing, LIU Yong-yang, HUANG Yong-ping. Fast ant colony algorithm for solving traveling salesman problem[J]. Journal of Jilin University (Engineering and Technology Edition),2013,43(01):147-151.

7. WANG Jianwen, DAI Guang-ming, XIE Bai-qiao, ZHANG Quan-yuan. A survey of solving the traveling salesman problem[J]. Computer Engineering \& Science,2008(02):72-74+155.

8. Dorigo M, Maniezzo V, Colorni A. The Ant System: Optimization by a Colony of Cooperating Agents[J].IEEE Transon Systems, Man, and Cybernetics Part B,1996,26(1):29-41.

9. Dorigo M, Gambardella L M. A Study of Some Properties of Ant-Q[C] // Proc of the 44th Int'l Conf on Parallel Problem Solving from Nature,1996: 656-665.

10. Thayer T C, Vougioukas S, Goldberg K, et al. Routing Algorithms for Robot Assisted Precision Irrigation[C]. international conference on robotics and automation, 2018: 2221-2228.

11. Tokekar P, Hook J V, Mulla D, et al. Sensor planning for a symbiotic UAV and UGV system for precision agriculture[C]// Intelligent Robots and Systems (IROS), 2013 IEEE/RSJ International Conference on. IEEE, 2013.

12. Aspects of Precision Agriculture S. T. Jawaid and S. L. Smith, "Informative path planning as a maximum traveling salesman problem with submodular rewards," Discrete Appl. Math., vol. 186, pp. 112-127, 2015.

13. T. H. Pham, Y. Bestaoui and S. Mammar, "Aerial robot coverage path planning approach with concave obstacles in precision agriculture," 2017 Workshop on Research, Education and Development of Unmanned Aerial Systems (RED-UAS), Linkoping, 2017, pp. 43-48.

14. Zhou K, Leck Jensen A, S Rensen C G, et al. Agricultural operations planning in fields with multiple obstacle areas[J]. Computers and Electronics in Agriculture, 2014, 109:12-22.

15. Han Yu. Study on genetic algorithm for travel salesman problem[D]. Southwest Jiaotong University,2006. 